\title{
Electrostatic charge measurement in hydraulic circuits
}

\author{
M. Kühnlein, T. Wendel and H. Murrenhoff \\ RWTH Aachen University, Institute for Fluid Power Drives and Controls (IFAS), Aachen, Germany \\ E-mail: Michael.Kuehnlein@ifas.rwth-aachen.de
}

\begin{abstract}
Electrostatic charging of hydraulic circuits is recently becoming an issue as low conducting hydraulic fluids are increasingly in use. Charge generation is more likely to accumulate and build up large voltages. These high charge accumulations are hazardous as they can damage system components such as filters, which can eventually lead to system failure. In order to develop appropriate counter measures, a precise knowledge of influencing parameters is strongly beneficial. In this paper the causes for charge generation are discussed. Furthermore, methods for charge measurement of hydraulic fluids are presented. As the researched measurement methods are considered inappropriate, a new charge measurement method is developed. The development based on the principles of Faraday-cup and Faraday-cage are discussed in detail. Moreover, the integration of the developed charge sensors into an also developed experimental setup is described. The test rig in combination with the charge sensor allows the quantitative measurement of charged fluid without influencing the fluid to be investigated. It also enables measurements under pressure, meaning that the fluid can be pressurised up to 140 bar. The experimental setup is destined to obtain a large data basis. This data basis allows the validation of a charging model for which the modelling approach is also presented in this paper. This will lay the basis for a charging model to predict the charging tendency of a hydraulic system.
\end{abstract}

Keywords: Electrostatic charging, electro-chemical double layer, charging of hydraulic fluids, flow through filters, charge measurement.

\section{Introduction}

Electrostatic charging is a well known phenomenon in many areas, one may think of experiencing a small flash of lightning while closing an automobile car door. An area of technical interest includes charging issues in electric power transformers of large scale, which were reported to explode due to electrostatic charging [1]. In these power transformers cooling fluid flows through long and small channels. The reason for electrostatic charging of fluids can be reduced to friction occurring between the flowing fluid and the wall. With unavoidable friction being present, electro-chemical double layers are built up at the wall [2] and the charges are separated by the flow of the liquid [3]. Thereby, charge is being accumulated. Charging of electric power transformers has been extensively analysed.

Charge accumulation is not challenging if the fluid is well conducting and the electric charge can be transported. Therefore, electrostatic charging has not been reported a major issue in hydraulic industry until recently. However, currently hydraulic oils with reduced ash and zinc concentrations are deployed to a greater extent [4]. These oils are less conducting and so lead to an increased charge accumulation.
As a result of the current trend towards low conducting hydraulic fluids, the topic of electrostatic charging becomes more important in the hydraulic industry. This paper gives an overview of the mechanisms responsible for charging and presents selected theories. Furthermore, a modelling approach for hydraulic systems is introduced. Another chapter deals with measurement principles and a new charge sensor, which was developed at the Institute for Fluid Power Drives and Controls (IFAS) of RWTH Aachen University. The presented measurement method is deployed in a hydraulic test circuit, which allows a detailed investigation of the phenomenon in order to expand the base of knowledge on electrostatic charging in hydraulic circuits.

In the later course of the project, for which some results are presented in this paper, a lot of experimental data will be generated. This data will serve as a knowledge base for the development of a charging model which should allow predicting the charging tendency of a hydraulic system. The current focus of the project is the investigation of the influence of hydraulic filters. Because of the large surface and its non-conducting material the filter element of a hydraulic circuit can naturally be regarded as the main source of charging. Until now, quantifiable data for hydraulic circuits regarding various influencing system 
parameters is not sufficient and the project under discussion is aimed to close this gap.

\section{Electrostatic charging of fluids}

The electrostatic charging process is mainly caused by two materials with differing work function being situated close together. Friction is not necessary but facilitates a small distance of the two partners [5]. Figure 1 illustrates the charging and its separation for solid bodies.
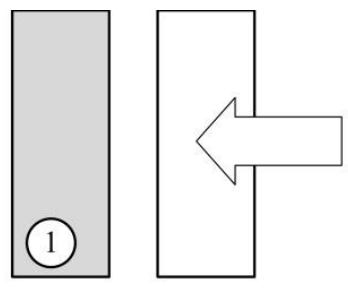

Contact

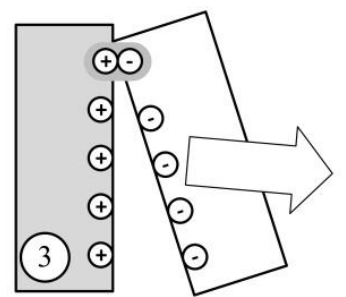

Partial charge balancing

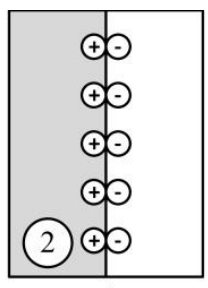

Distance: $\leq 10 \mathrm{~nm}$
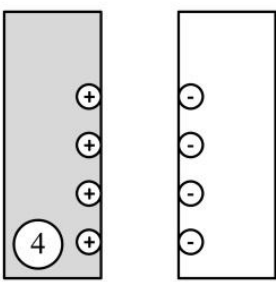

Electrostatic charged bodies
Figure 1: Charge separation for rigid bodies, based on [5]

If two bodies with different work functions are moved together (1) and the distance of the two bodies is less than $10 \mathrm{~nm}$ (2) electrons move from the material with the lesser work function towards the material with the higher work function. The reason for the charge transfer is the potential difference. An electrical double-layer builds up and the potential is in the range of millivolts. If the bodies are separated (3) the charge quantity stays constant. During the separation process the capacity of the setup decreases and so the previously low voltage can reach a voltage in the range of kilovolts (4). The duration of the separation process has a major influence on the potential generated. In general, a fast separation process leads to a high voltage, a slow separation process allows for charge compensation processes to take place so that only low voltage is generated.

For explanation of the charging process in fluids Figure 2 is introduced, which shows oil in a pipe.
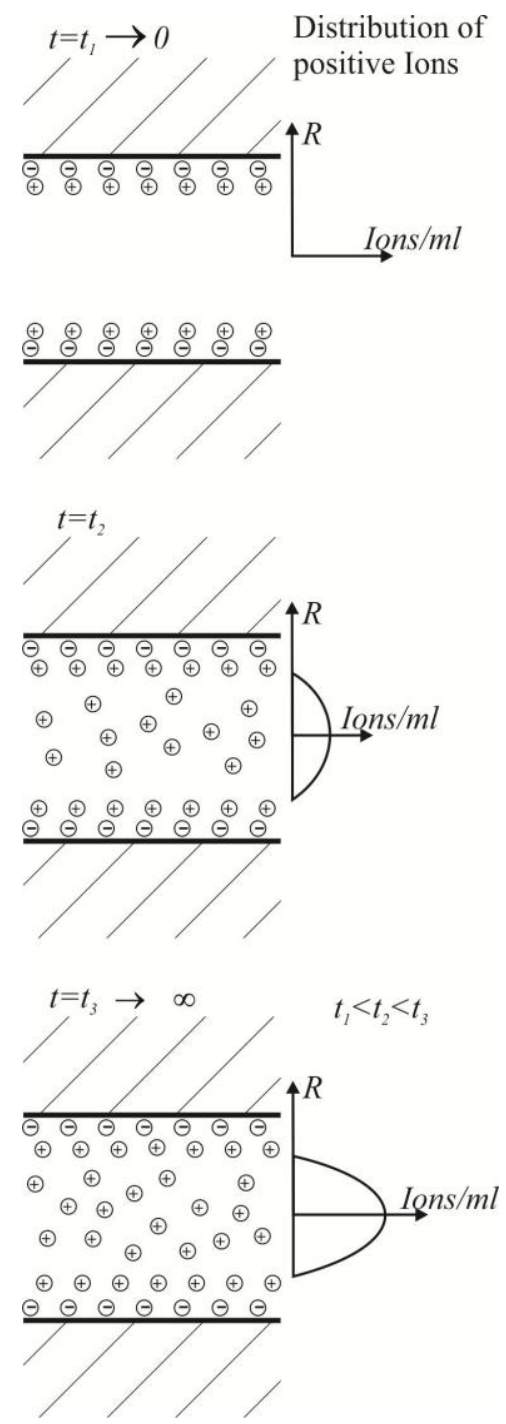

Figure 2: Electrostatic charging process

The charge separation takes place at the Helmholtz double layer [2]. The double layer builds up in the surface area of the wall. Assuming non-flowing fluid, negative ions adsorb on the boundary layer between pipe and oil on the pipe wall. For equilibrium reasons positive ions are in the adjacent layer. Hydraulic fluids are charged positively in most cases [6]. If the fluid flows through the pipe positive ions are carried away from the double layer and agglomerate in the fluid. With increasing time and volume flow a higher charge density is reached in the fluid.

Besides this basic flow electrification mechanism, more sophisticated flow electrification models have been developed. One of these is the nonlinear corrosion doublelayer model shown in Figure 3. 


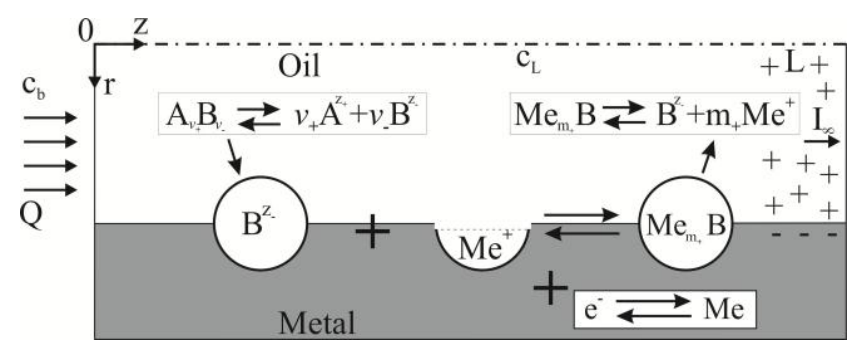

Figure 3: Corrosion double-layer model [3]

In this theory, oil has neutral molecules $\mathrm{AB}$ which dissociate into ions $\mathrm{A}^{+}$and $\mathrm{B}^{-}$. The $\mathrm{B}^{-}$ions mainly adsorb at the metal surface and start the metal corrosion with positive $\mathrm{Me}^{+}$ions. The MeB molecules are electrically neutral. As the $\mathrm{B}^{-}$ions are thereby neutralised, the positive $\mathrm{A}^{+}$ions remain in the fluid and thus charge the fluid positively [3].

Another theory focusses on the interaction between a cellulose material, also used in hydraulic filters, and oil. A description of the model shall be attained with Figure 4.

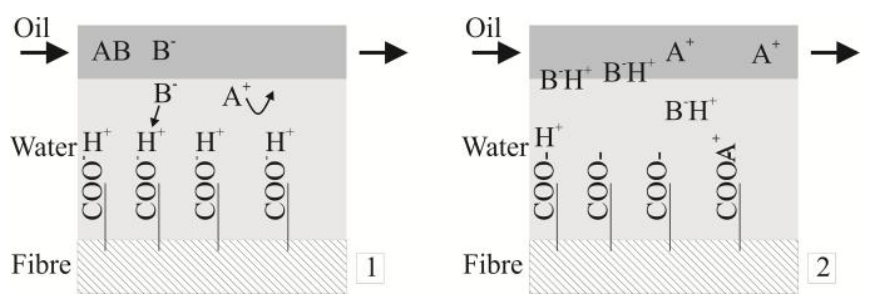

Figure 4: Cellulose fibre theory [7]

The theory states that the fibre structure has carboxy groups $\mathrm{COOH}$ in its boundary and the fibre surface is covered by a thin water layer. In the oil there are neutral molecules $\mathrm{AB}$ which can dissociate into $\mathrm{A}^{+}$ions and $\mathrm{B}^{-}$ions. The $\mathrm{A}^{+}$ions are assumed to have a lower mobility than the $\mathrm{B}^{-}$ions. Therefore, the mobile $\mathrm{B}^{-}$ions combine with the $\mathrm{H}^{+}$ions of the carboxy groups and constitute the neutral molecule $\mathrm{BH}$. This leads to a neutralisation of the negative ions and so the oil is charged positively with $\mathrm{A}^{+}$ions.

The project discussed in this paper has a system view on electrostatic charging. Therefore, the whole charge caused by the system is being considered. To allow a prediction of the charging tendency of a hydraulic system, a charging model is set up. The model will be based upon the data obtained later within the course of the project. As a system consists of many components, the charging model is formulated as a charging model of individual components or system parts. As the focus of the project is on hydraulic filters, the charge $Q_{\text {Filter }}^{\text {el }}$ caused by filters is considered vital. Charge $Q_{\text {Pipe }}^{\text {el }}$ caused by pipes is another part of the model. These individual "sub-charges" are summed up to yield the overall charge $Q_{0 \text { verall }}^{\text {el }}$ of the system. The factors $P_{\text {Pipe }}$ and $P_{\text {Filter }}$ are utilised as weighting factors, to account for different pipe length and various filter sizes.

$$
\begin{aligned}
Q_{\text {Overall }}^{\text {el }}=Q_{\text {Pipe }}^{\text {el }} P_{\text {Pipe }} & \sum_{i=1}^{n} K_{i, \text { Pipe }} \\
& +Q_{\text {Filter }}^{\text {el }} P_{\text {Filter }} \sum_{i=1}^{m} K_{i, \text { Filter }}
\end{aligned}
$$

The sub-charge $Q_{\text {Pipe }}^{\text {el }}$ will be predicted with a charging model for pipes. The model from Klinkenberg seems promising, as it distinguishes between laminar flow and turbulent flow [1]. For the charge caused by the filter $Q_{\text {Filter }}^{\mathrm{el}}$ the electrification model from Washabaugh seems appropriate [8]. This model describes the flow through the area between two pipes where the outer pipe is metal and the inner pipe is non-conducting. In Equation (1) there are $K_{\mathrm{i}}$ factors included, which are used to describe the dependency of the charging quantity on influencing factors such as volume flow, temperature, particle contamination, filter material and so on. If for example the volume flow is doubled and the resulting charge quantity is twice as high as the charge quantity obtained with the standard parameter set, the corresponding $K_{\mathrm{i}}$ factor is 2 . However, the sub-models which can be applied will be determined as soon as measurement data is available.

As the charging process and the modelling approach have been discussed, the question arises how the charge could be measured in an experimental setup. Considered measurement methods and the development of a charge sensor meeting the requirements of the challenging project are presented in the next chapter.

\section{Measurement methods}

In electrostatics, the physical value voltage cannot be measured with classical measurement instruments. This is due to the fact, that in electrostatic systems there is no constant voltage source and the amount of charges is limited. Therefore, special attention needs to be paid to isolation in order not to remove any charges from the object to be measured.

In Figure 5 electrostatic voltmeters are outlined, which have a very high resistance and despite that an extremely low tendency to discharge metal parts. Both instruments are strictly mechanical and utilise the force action of electric fields on charge carriers. The instrument shown on the left is basically a parallel-plate capacitor and the distance of the field plates changes depending on the applied voltage and the return spring. A scale allows the quantification of the measured voltage. The instrument shown on the right is an angle dependent rotary capacitor; the measured voltage is in linear relation to the torque $M$. 

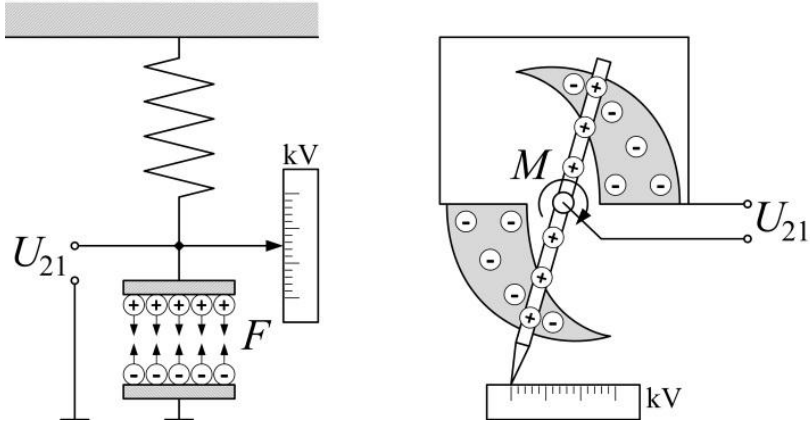

Figure 5: Electrostatic voltmeters, adapted from [5] and [9]

Figure 6 shows another instrument, which allows the measurement of electric fields. A grounded impeller wheel rotates with constant speed above fixed induction electrodes.

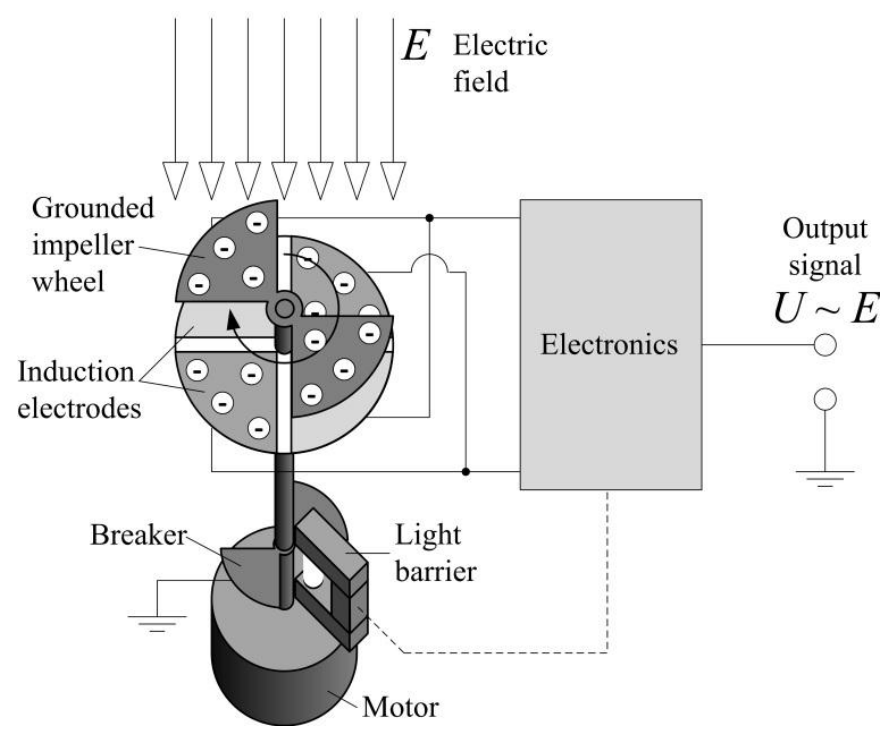

Figure 6: Electro field meter, adapted from [10] and [11]

As an electric field is present, the impeller wheel either exposes or shields the induction electrodes and with amplifying and conditioning electronics the output voltage $U$ is proportional to the field strength $E$. If the electro field meter shown in Figure 6 is used with the voltage measuring head illustrated in Figure 7 voltages can be measured. The voltage measuring head is mounted to the electro field meter and adds a field plate, so that a parallel-plate capacitor is build. The object to be measured is connected to the measurement socket and the charge on the field plate causes an electric field between field plate and the induction electrodes. The grounded impeller wheel rotates in-between field plate and induction electrodes.

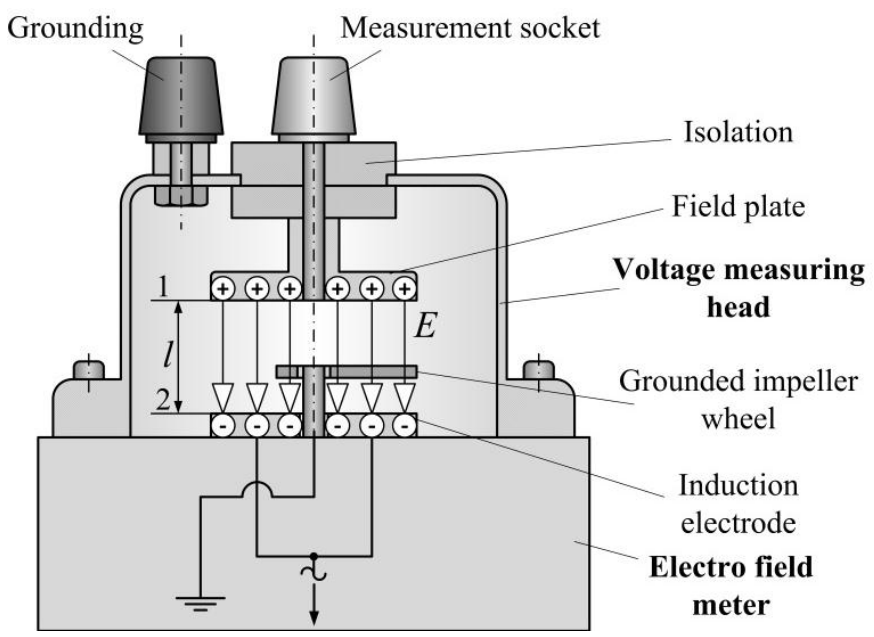

Figure 7: Voltage measuring head, adapted from [5]

For a homogeneous electric field the voltage $U_{21}$ is a product of the field strength $E$ and the distance $l$.

$$
U_{21}=E l
$$

The distance $l$ in the voltage measuring head is precisely known and the field strength $E$ is measured with the electro field meter, so the voltage of any object connected to the measurement socket can be measured. As the combination of electro field meter and voltage measuring head are in principal a plate capacitor, the isolation against discharge as well as the accuracy is very high. It therefore qualifies as an appropriate method to measure the voltage.

\subsection{Measuring charges in fluids}

To investigate the electrostatic charge of fluids, some methods have been developed in the past. The most relevant of these are presented in short in this chapter.

Figure 8 shows the Mini-Static Tester, which was initially developed by Oomen [12] to allow a detailed investigation of transformer oils.

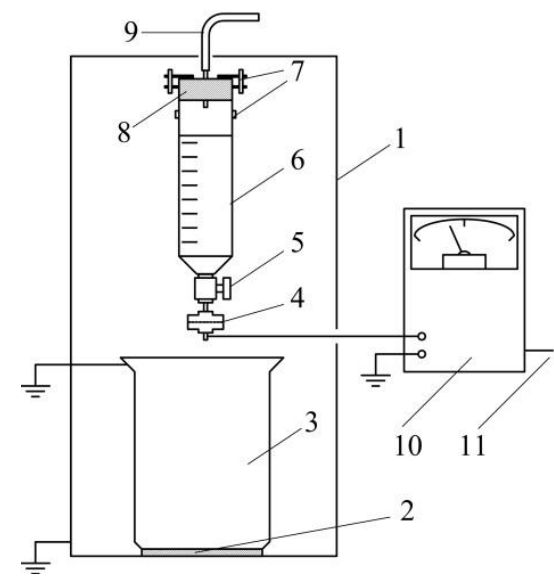

Faraday-cage

2 Isolation plate

3 Metal cup

4 Filter holder

5 Valve

6 Plastic syringe

Mounting brackets

8 Plug

9 Pressure line

10 Electrometer

11 Signal output

Figure 8: Mini-Static Tester, adapted from [12] 
An oil test volume is pressed through a filter (4), where a charge separation takes place. A metal cup (3) collects the filtered oil and the charging current of the filter holder is detected with an electrometer (10). The amount of charge transferred to the filter holder is equivalent to the amount of charge generated in the fluid [12].

With consideration of the volume flow the volume charge density $\eta$ can be calculated from the charging current measurement. Later, improvements have been made to the Mini-Static Tester in Japan, where the plastic syringe has been replaced by a glass container and a heating system was added. Furthermore, the charging current was measured at the metal cup instead of the filter holder. The volume charge density was then determined from the difference of a measurement with filter material and a measurement without filter material. Thereby, the non-identifiable influence of the cup on the charging process is omitted [13]. The Mini-Static Tester is not appropriate for measurements under higher pressure and the results obtained are charging tendency instead of absolute values.

The Absolute-Charge-Sensor (ACS) uses the Faraday-cup principle, see Figure 9.

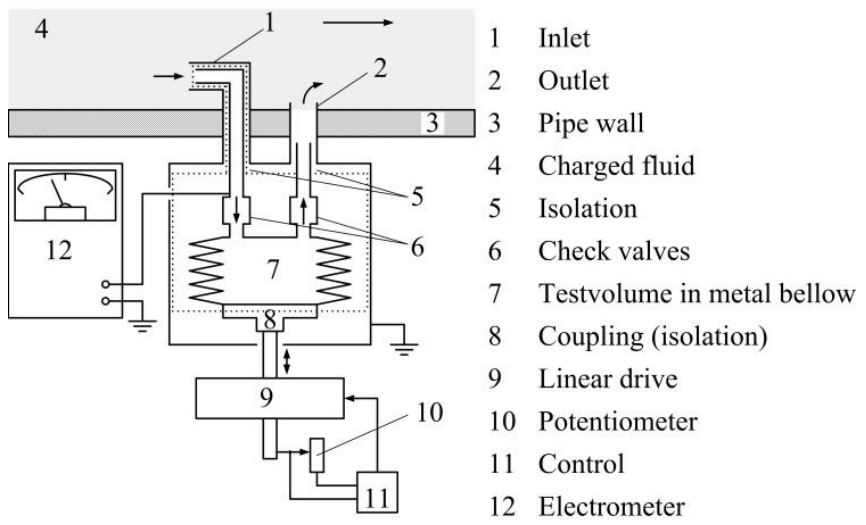

Figure 9: Absolute-Charge-Sensor, adapted from [14]

The charged fluid flows into a metal bellow (7), and the volume of the bellow can be altered with a linear drive (9). By means of influence the charge of the fluid causes a charge on the outside of the metallic bellow. This charge is measured with an electrometer (12). A grounded housing shields the instrument from surrounding electric fields. The volume charge density is calculated from the measured current and the fluid volume flow [14]. The AbsoluteCharge-Sensor (ACS) is not suitable for measurements at higher pressure, as the material strength of the metal bellow is limited.

Another method to determine the volume charge density is the Tandem-chamber Charge Density Monitor shown in Figure 10. It uses the relaxation behavior of the electrostatically charged fluid and consists of two identical measurement chambers (5), the housings of the measurement chambers are separated by an isolating flange (6).

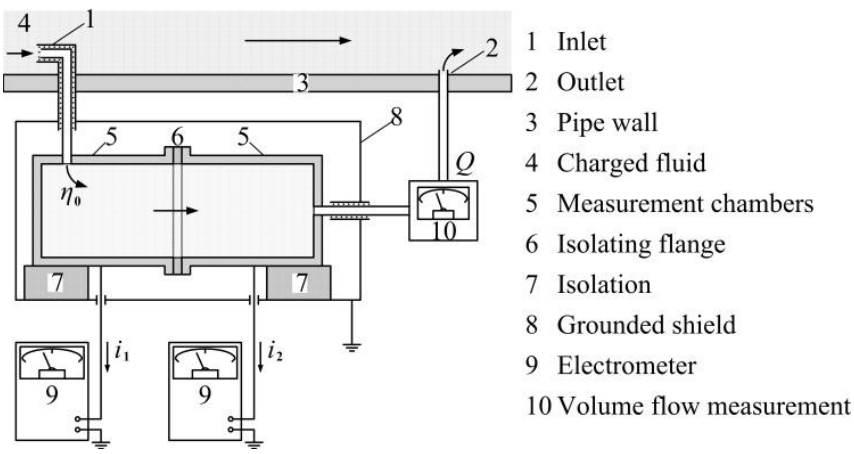

\section{Figure 10: Tandem-chamber Charge Density Monitor, adapted from [15]}

The volume charge density is approximated from the differential current measurement of the two electrometers (9), taking the volume flow into account. The Tandemchamber Charge Density Monitor is not suitable, if higher volume flows are to be investigated, as charges could be dragged from the first chamber to the second. Furthermore, it is very likely to be affected by perturbations.

None of the researched possible measurement principles are considered suitable to investigate charged fluid in a hydraulic system, either because of only low pressure applicability or questionable repeatability due to perturbation affection. Therefore, a new measurement method, which can easily be integrated into hydraulic circuits, needs to be developed. This development is described in the next chapter.

\subsection{Development of a charge sensor}

The main challenge of the required charge sensor is that no hydraulic pipe should be opened to extract charged fluid. This implies the need of a contact-free measurement principle. As charged fluid is surrounded by an electric field, this is considered a starting point. By integrating the electric field strength $\mathrm{E}$ over its enveloping area $\mathrm{A}$, the charge quantity q on the enveloping area can be calculated [9]:

$$
\iint_{A} \varepsilon_{0} \varepsilon_{r} \boldsymbol{E} d \boldsymbol{A}=q
$$

This equation is based on Maxwell's equation on enveloping flow. The electrical field constant $\varepsilon_{0}$ is a natural constant and has a value of $8.845210^{-12} \mathrm{As} /(\mathrm{Vm}) . \quad \varepsilon_{\mathrm{r}}$ is the dimension-free relative permittivity, which is material dependent and always greater than zero. Equation (3) shows, that if the field strength $E$ is known on every point of the enveloping area $A$, the enveloping charge quantity can be derived without a physical contact with the charged fluid. Thinking in practical terms, it would allow a direct detection of the charged fluid, even if the fluid is pressurised. Furthermore, no charge would be withdrawn and an accurate measurement could be carried out. The field strength could be measured with the electro field meter described in chapter 3.1 . 
The determination of the electric field could prove to be challenging, as in reality there are always interfering fields which influence the measurement. Moreover, the measured field strength depends on the localisation of the measurement. However, if one considers a Faraday-cup, the charge is equally distributed on the cup surface and thereby the electric field is also equally distributed. As an electrically conducting cup is an equipotential surface, the electric field lines are perpendicular with regard to the surface. Then the vector product $\boldsymbol{E} \mathrm{d} \boldsymbol{A}$ from Equation (3) simplifies to a scalar product $E \mathrm{~d} A$. If the relative permittivity is constant it follows:

$$
\varepsilon_{0} \varepsilon_{r} E \iint_{A} d A=q
$$

For surface geometries which are mathematically easy to describe, it is possible to give the charge quantity enclosed in a Faraday-cup if the electric field strength at one point of the surface and the relative permittivity are known.

To account for interfering fields which would perturb the measurement, an electrical capacitor could be used. Capacitors with a simple geometry allow an analytical determination of the charge density and thus the field strength.

The ideal solution therefore would be a combination of a Faraday-cup and a capacitor. Such a combination is drafted in Figure 11.

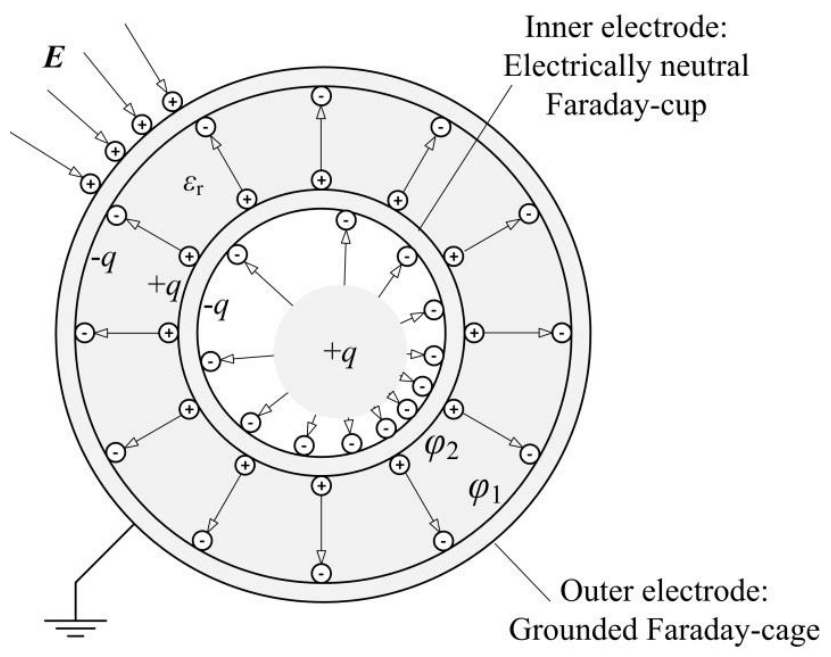

\section{Figure 11: Electrical capacitor as a combination of a Faraday-cup and a Faraday-cage}

The Faraday-cup itself is electrically neutral, meaning that the quantity of negative charge on its inner surface equals the quantity of positive charge on its outer surface. The neutrality is always prevalent. If a charge $+q$ is present in the middle of the cup, an electric field builds up and consequently the inner surface of the cup holds a negative charge. Caused by influence, positive charge is moved to the outer surface of the cup. This causes an electric field between cup and outer electrode. Depending on the field weakening influence of the dielectric, a potential difference
$U=\varphi_{1}-\varphi_{2}$ develops between the outer surface of the cup and the inner surface of the outer electrode. From the potential difference $U$ the charge quantity $q$ can be calculated if the capacity $C$ is known.

$$
q=C U
$$

Another possibility is the measurement of the charge. The charge could be conducted from the Faraday-cup and the conducting current could be measured. This method would have the disadvantage, that the Faraday-cup would not be electrically neutral after the measurement. Another possibility is the measurement of the field strength within the capacitor. This would necessitate inserting the measuring device into the capacitor and thereby influencing the measurement itself.

The most promising approach is to measure the potential difference $U=\varphi_{1}-\varphi_{2}$ and calculate the charge quantity with Equation (5). The potential difference can be measured with the voltage measuring head in combination with an electro field meter shown in Figure 7. Advantages are that the capacitor setup is not perturbed by inserting a measuring device and the Faraday-cup can be kept electrically neutral.

Considering the test rig, a cylindrical capacitor is best suited because the charged fluid can be easily brought into the capacitor. Figure 12 shows a sectional view of a cylindrical capacitor with charged fluid inside.

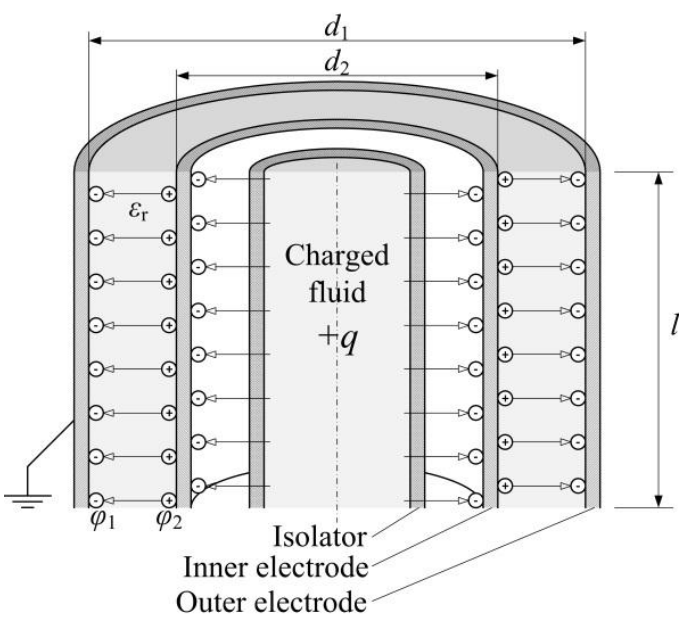

Figure 12: Sectional view of a charged cylindrical capacitor

The electric field caused by the charged fluid $(+q)$ leads to a charge movement on the inner electrode. On the inside surface of the inner electrode negative charge accumulates. As the inner electrode is electrically neutral, positive charge accumulates on its outer surface which is the potential $\varphi_{2}$. Charged fluid and inner electrode are aligned collinear so that the field lines are perpendicular to the fluid and impinge on the inner electrode without deflection. The whole displacement current caused by a fluid column with the length $l$ is taken up by the inner electrode of the same length. Field deflections in the edge areas of a practical cylinder capacitor can be reduced to a minimum by a proper design. 
The outer electrode is grounded and so shields the capacitor against surrounding interfering fields. The potential $\varphi_{2}$, which for the grounded outer electrode is equal to the potential difference $U$, is measured without charge loss with the voltage measuring head. The volume $V$ holding the fluid charge quantity is given by the geometric properties of the fluid column.

As the volume $V$ is known, the mean charge density $\eta$ can be determined.

$$
\eta=\frac{q}{V}=\frac{C U}{V}
$$

The capacity $C$ of a cylindrical capacitor, where $d_{2}$ is the outer diameter of the inner electrode and $d_{1}$ is the inner diameter of the outer electrode, is:

$$
C=\frac{2 \pi \varepsilon_{0} \varepsilon_{r} l}{\ln \left(d_{2} / d_{1}\right)}
$$

Inserting Equation (7) into (6) yields:

$$
\eta=\frac{2 \pi \varepsilon_{0} \varepsilon_{r} l}{V \ln \left(d_{2} / d_{1}\right)} U
$$

To calculate the mean charge density $\eta$ the relative permittivity $\varepsilon_{r}$ of the medium in the capacitor needs to be known. As a medium either air or an isolation gas could be used, for which the permittivity can be found in literature or can be calculated [16].

For dimensioning it is important to specify the parameters. The chosen voltage measuring head has a maximum detectable voltage of $2000 \mathrm{~V}$. The volume $V$ of the charged fluid is given by:

$$
V=\frac{d_{f l}^{2} \pi}{4} l
$$

Inserting Equation (9) into (8) yields:

$$
\eta=\frac{2 \pi \varepsilon_{0} \varepsilon_{r}}{\frac{d_{f l}^{2} \pi}{4} \ln \left(d_{2} / d_{1}\right)} U
$$

It is clear, that the charge density $\eta$ does not depend on the length of the fluid column. Sizing parameters are the diameter of the fluid column $d_{\mathrm{fl}}$, and the electrode diameters $d_{1}$ and $d_{2}$. As a fluid column a non-conducting hydraulic hose is used, which has an inner diameter $d_{\mathrm{fl}}$ of $25.4 \mathrm{~mm}$. In order to allow a dimensioning of the electrode diameters, it is necessary to estimate the expected charge density. Data for the charge density is hard to find. From Sasaki's data a mean charge density of $2.710^{-3} \mathrm{C} / \mathrm{m}^{3}$ can be derived [6]. However, the measuring method of Sasaki is different and the stated value is calculated from transformer oil data where hydraulic mineral oil should be investigated in the case discussed in this paper. The "Technical Rules for Operation Safety" states values of $5.010^{-3} \mathrm{C} / \mathrm{m}^{3}$ as typical values of the charge density downstream of micro filters [17]. The circumstances leading to these values are not further specified. As no other relevant data is available, the data from Sasaki is considered most applicable.
To account for unexpected higher charging, a 20\% safety margin is added so that the capacitor is dimensioned for a measuring range of $3.2410^{-3} \mathrm{C} / \mathrm{m}^{3}$. An outer diameter $d_{2}$ of the inner electrode of $160 \mathrm{~mm}$ is chosen and so the inner diameter $d_{1}$ of the outer electrode, under consideration of Equation (10), is $171 \mathrm{~mm}$.

A simplified view of the measurement setup is shown in Figure 13. If one considers the test procedure another obstacle is to be overcome. The charged fluid does not necessarily loose its charge after the measurement and therefore an electric field would still be present. If the inner electrode was grounded beforehand of each measurement, it would immediately assume the charge according to the prevalent electric field. This would falsify the measurement because the initial potential would not be zero. Therefore, a shielding pipe is added to the setup. As the shielding pipe is grounded, the inner electrode is shielded on either side and is in a field-free space.

In this field-free space the inner electrode can be grounded in it assumes zero potential. The measurement procedure is as follows:

1. Insert shielding pipe

2. Shortly ground inner electrode

3. Expose charged oil to capacitor

4. Remove shielding pipe

5. Start charge measurement

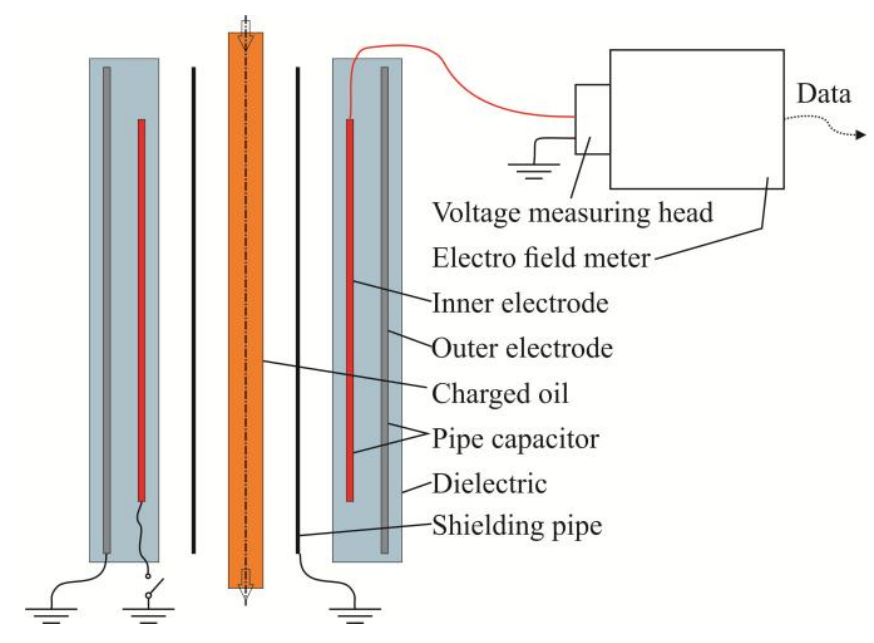

Figure 13: Simplified view of measurement setup

The developed sensor allows a measurement without a loss of charge and does not influence the fluid to be measured. It can easily be integrated into hydraulic circuits and enables measurements under pressure up to 140 bar.

The length $l$ of the capacitor is set to $200 \mathrm{~mm}$, which is useful in practical terms. Thereby, supplemental electrodes can be added at each end of the inner electrode to minimise deflections of the field lines at both ends of the inner electrode. A sectional view of the developed measurement capacitor is depicted in Figure 14. 

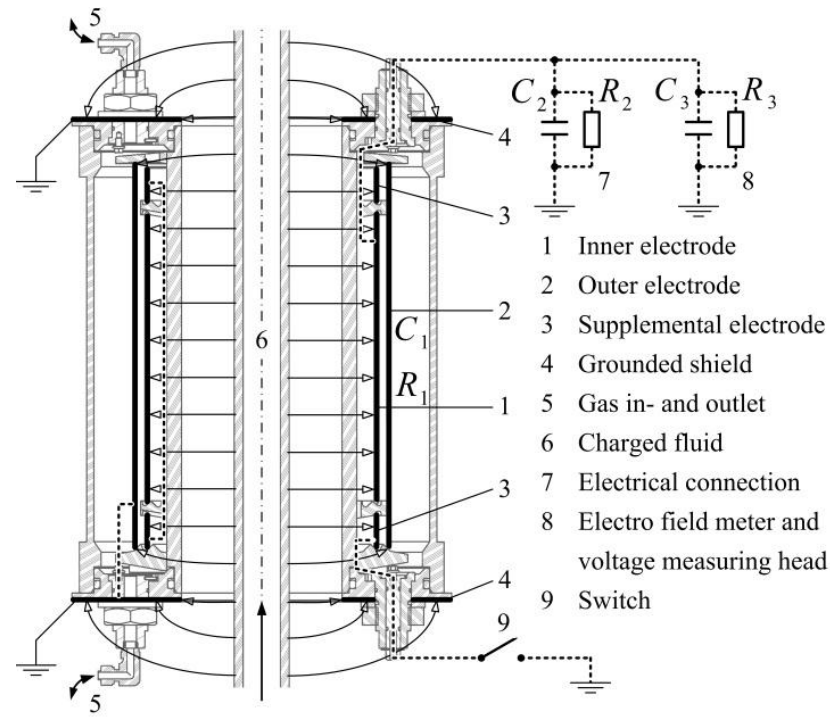

Figure 14: Measurement capacitor

The whole capacitor consisting of the inner electrode, the outer electrode and the two supplemental electrodes is enclosed in isolating polytetrafluoroethylene (PTFE). Thereby, in can be surrounded by a gaseous dielectric. The inner electrode is connected to the voltage measuring head. Outer electrode and supplemental electrodes are grounded during measurements.

The next chapter shows the test rig for the investigation of charged hydraulic fluids caused by filters.

\section{Test rig}

The hydraulic schematic of the test rig is shown in Figure 15. It consists of a filter module. Pumps provide the oil flow through the filter and the system pressure can be set by a pressure control valve to modify the load. The volume flow of the pumps is $71 / \mathrm{min}$ or $21 \mathrm{l} / \mathrm{min}$.

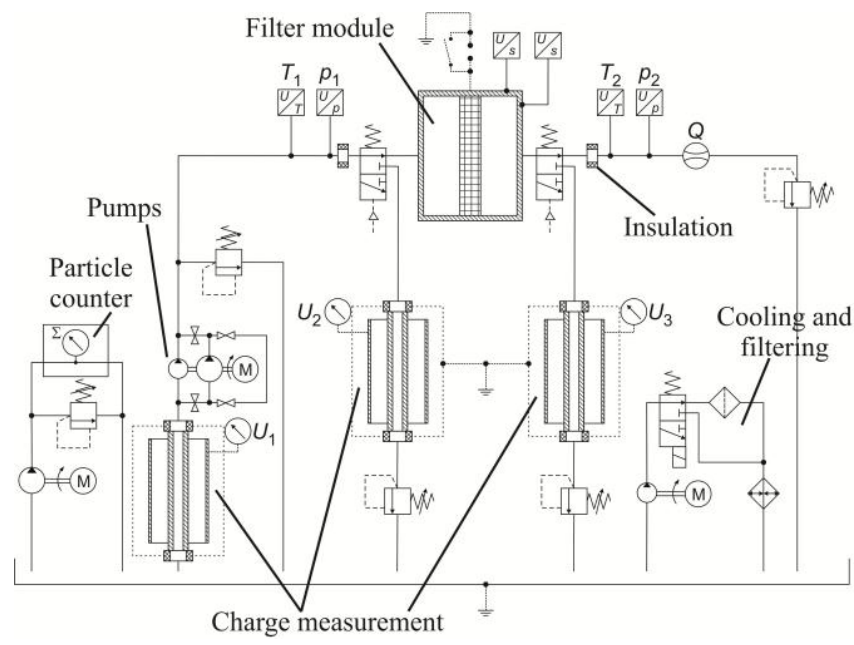

Figure 15: Test rig schematic

Besides the filter, the charge measurement chambers are the core elements of the test rig. These chambers allow the charge measuring of the oil. The fluid is measured before it flows through the filter and after it has passed the filter module. The charge caused by the filter module is calculated as a difference of up- and downstream measurement. The measurements are carried out intermittent and the necessary flow is taken from the main circuit with pneumatically operated ball valves. A third charge measurement chamber is situated upstream of the pumps to analyse the initial charge of the fluid after leaving the reservoir. Additionally, a cooling and system-filtering sub-circuit is included. A particle counter allows maintaining defined oil cleanliness. To be able to investigate the filter element housing grounded and ungrounded, the housing is completely insulated and can be electrically grounded on purpose.

The physical test rig is shown in Figure 16. The shielding pipe, as a part of the measurement chambers, needs to be inserted and removed during each measurement cycle. To ensure comparable tests, this motion is automated pneumatically. The oil tank holds a volume of 2501 , which accounts for a long relaxation time so the charge should be zero when the oil leaves the tank. To maximise air separation, design recommendations from Weimann are accounted for [18]. The tank is equipped with a spiralshaped insert which increases the surface area and undissolved air bubbles are expected to rise faster to the surface.

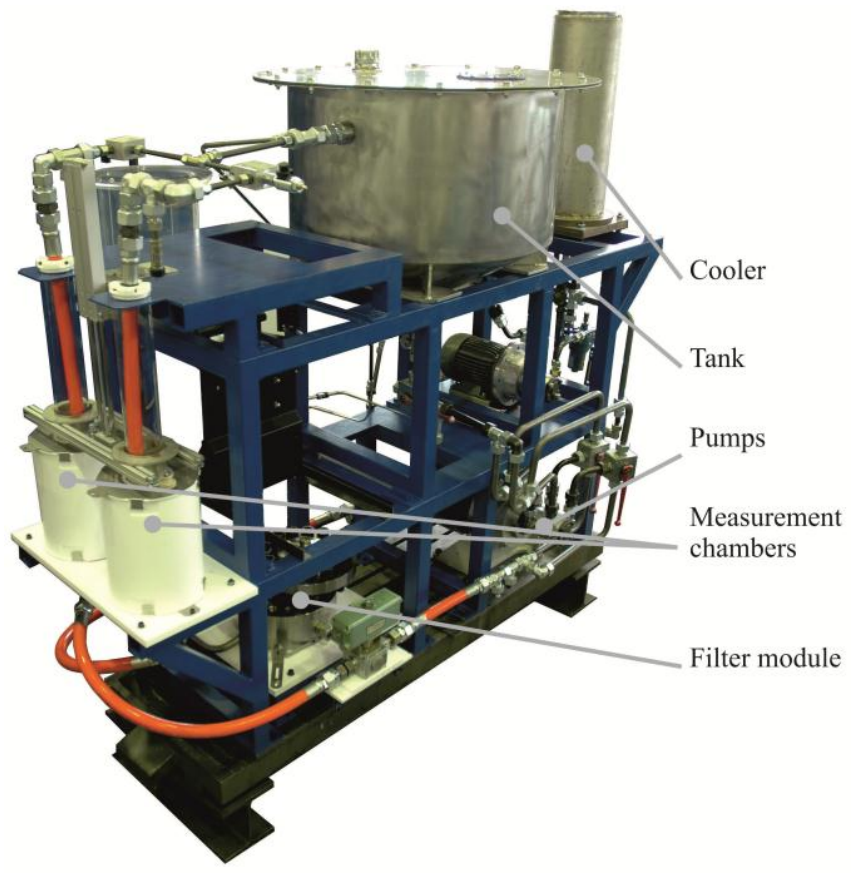

Figure 16: Test rig

Furthermore, the tank is designed cylindrically with a cone shaped bottom to prevent particles from being sedimented at the tank bottom. Another feature is that the test rig is remotely controllable. This is an advantage as the test rig is to be operated under varying temperature conditions.

A detailed view of the filter element housing is shown in Figure 17. The filter material is chosen to be circular and flat in shape to avoid geometry influences on the charge measurement. The filter disk is clamped in the middle of the shown housing. The housing consists of two parts and is connected by screws, which have been dimensioned so that 
the housing withstands 350 bar. The dimensioning to 350 bar was verified by simulation and chosen, so that the filter module could also be operated in different hydraulic systems.
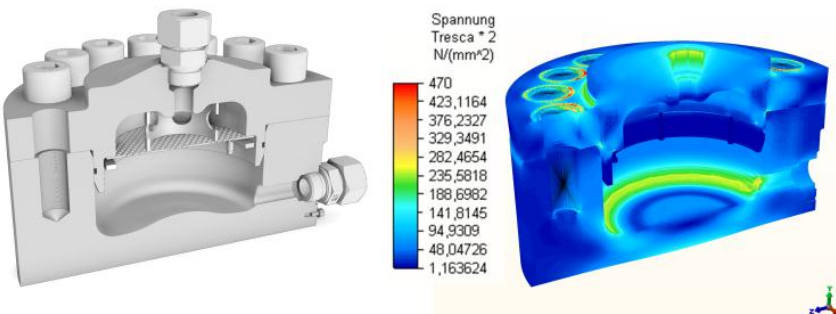

Figure 17: Filter element housing for simplified filter geometries

To allow an optimised and uniform flow through the filter material and account for a sturdy design, the bottom of the housing is designed with an elevation in its middle. As has been pointed out, the filter material is flat in shape. To investigate the influence of the filter material on the charging effect, three different filter media are used as a start. These media are shown in Figure 18.

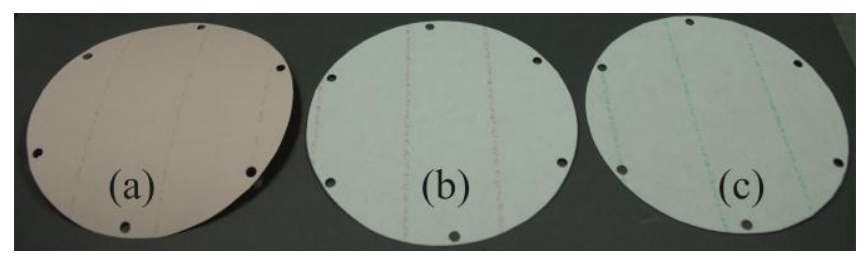

Figure 18: Filter media for filter module

Material (a) is cellulose and has a typical pore size of $15 \mu \mathrm{m}$, material (b) is glass fibre with a pore size of $6 \mu \mathrm{m}$ (according to standard ASTM E 1294) and material (c) is also glass fibre with a typical pore size of $30 \mu \mathrm{m}$. The cellulose media is first investigated, as it will allow the validation of the developed charging model. The glass fibre media are investigated as they are closer to filter products widely used in hydraulic industry.

Other parameters to be investigated in the project are the oil temperature, the oil pressure, the oil conductivity, the influence of particles, the water content of the oil, grounded and ungrounded filter housing and volume flow dependency. As this given first parameter set would lead to approximately 30000 single tests which need to repeatedly measured, a Design of Experiments method (DOE) has been applied to reduce the number of experiments because this huge amount of tests could not be realised. Thereby, a first screening test was set up which necessitates 64 experiments. After the first results, a new test schedule based on the experiences can be set up.

The test rig has another feature with regard to safety. The filter module can be investigated if ungrounded and is therefore electrically insulated from the other components. The "Technical Rules for Operation Safety" give limit values to hinder electrical discharges, which are a danger for persons. An energy content $W$ of $350 \mathrm{~mJ}$ and a charge quantity $q$ of $5010^{-6} \mathrm{C}$ should never be exceeded [17]. To account for the safety of persons, a spark gap is designed to the filter module (see Figure 19).

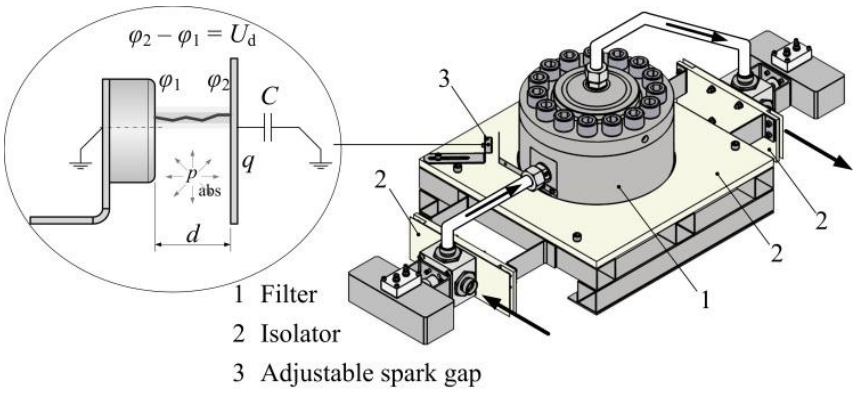

Figure 19: Adjustable spark gap at filter module

With the adjustable spark gap the distance is chosen in such a way, that a discharge occurs before the limit values are reached. The required distance can be calculated based on Paschen's law [9]:

$$
U_{\mathrm{d}}=\frac{B_{\mathrm{g}} p_{\mathrm{abs}} d}{\ln \left(\frac{A_{\mathrm{g}} p_{\mathrm{abs}} d}{\ln (1+1 / \gamma)}\right)}
$$

It is an analytical approximation equation for the disruptive voltage $U_{\mathrm{d}}$ in ideal plate capacitors. $A_{\mathrm{g}}$ and $B_{\mathrm{g}}$ are gas constants, which have values of $11301 /(\mathrm{mm} \mathrm{bar})$ and $27.4 \mathrm{kV} /(\mathrm{mm}$ bar) respectively for air at a pressure of $1 \mathrm{bar}$ and at room temperature. The coefficient $\gamma$ for air and steel is $210^{-6} 1 /(\mathrm{mm}$ bar) [9].

The energy $W$ held by a capacitor is:

$$
W=\frac{1}{2} C U^{2}
$$

With the limit of $350 \mathrm{~mJ}$ given by [17] and the known capacity of the filter module housing, the distance $d$ to be set can be determined. Depending on the capacity, a typical value for the distance $d$ is $8 \mathrm{~mm}$. In a next step the capacity will be measured and so the distance can be set.

\section{Conclusion}

Electrostatic charging is a phenomenon recently occurring more often in hydraulic systems due to hydraulic fluids of low conductivity being applied more widely. As experimental data on the subject is hard to find, aim of the project presented in this article is acquiring such data and developing a model which allows the determination of the charging tendency of a hydraulic system.

The paper discusses the reasons for charging of fluids, which are the build-up of an electrochemical double-layer. If a flow is present, ions are removed from the double-layer and the fluid is charged.

To measure the charged fluid a suitable measurement method is in need. As the researched measurement methods are not considered appropriate, a new charge sensor is developed. It was shown that the electric field of a charged fluid column can be used as a basis for the measurement. A combination of a Faraday-cup and a Faraday-cage were developed and designed as a pipe capacitor. To measure the 
charge of the inner measuring electrode an electro field meter in combination with a voltage measuring head was chosen. The advantage is that the voltage of objects can nearly be measured without a loss of charge and thereby preventing a measurement failure. Furthermore, the charge of the fluid is not affected by the measurement.

The whole charge sensor, consisting of the pipe capacitor, the electro field meter and the voltage measuring head, was incorporated into a test setup. The developed experimental setup has a test filter module to investigate various filter types and materials. The fluid is measured up- and downstream of the filter module and by building the difference of the measured charge the influence of the hydraulic filter can be determined. The filter housing design and the safety precautions taken to avoid danger for persons, namely an adjustable spark gap, have been described.

Future work includes the start-up of the developed test rig to obtain the desired experimental data. After data acquisition the focus will be on a further specification and validation of the charging model.

\section{Acknowledgement}

The work and results presented in this article were achieved within the project "Untersuchung der elektrostatischen Aufladung von Hydraulikflüssigkeiten bei der Durchströmung von Filtern", funded by the German Alliance for Industrial Research (AiF) under grant number 17323/N1.

\section{Nomenclature}

\begin{tabular}{lll}
\hline Designation & Denotation & Unit \\
\hline$A$ & Area & {$\left[\mathrm{mm}^{2}\right]$} \\
\hline$A_{\mathrm{g}}$ & Gas constant & {$[1 /(\mathrm{mm}$ bar $)]$} \\
\hline$B_{\mathrm{g}}$ & Gas constant & {$[\mathrm{kV} /(\mathrm{mm}$ bar $)]$} \\
\hline$C$ & Capacity & {$[\mathrm{F}]$} \\
\hline$d$ & Diameter & {$[\mathrm{mm}]$} \\
\hline$d_{\mathrm{fl}}$ & Fluid column diameter & {$[\mathrm{mm}]$} \\
\hline$d_{1}, d_{2}$ & Diameters & {$[\mathrm{mm}]$} \\
\hline$E$ & Field strength & {$[\mathrm{V} / \mathrm{m}]$} \\
\hline$K_{\mathrm{i}}$ & Fitting factors & {$[-]$} \\
\hline$l$ & Length & {$[\mathrm{mm}]$} \\
\hline$M$ & Torque & {$[\mathrm{Nm}]$} \\
\hline$p_{\text {abs }}$ & Pressure & {$[\mathrm{bar}]$} \\
\hline$P_{\text {Filter }}$ & Weighing factor filter & {$[-]$} \\
\hline$P_{\text {Pipe }}$ & Weighing factor pipe & {$[-]$} \\
\hline$q$ & Charge & {$[\mathrm{C}]$} \\
\hline$Q^{\mathrm{el}}$ & Charge & {$[\mathrm{C}]$} \\
\hline$U, U_{21}, U_{\mathrm{d}}$ & Voltage & {$[\mathrm{V}]$} \\
\hline
\end{tabular}

\begin{tabular}{lll}
\hline$V$ & Volume & {$\left[\mathrm{mm}^{3}\right]$} \\
\hline$W$ & Work & {$[\mathrm{J}]$} \\
\hline$\gamma$ & Gas and material constant & {$[1 /(\mathrm{mm}$ bar $)]$} \\
\hline$\varepsilon_{\mathrm{r}}$ & Relative permittivity & {$[-]$} \\
\hline$\varepsilon_{0}$ & Permittivity & {$[\mathrm{A} \mathrm{s} /(\mathrm{V} \mathrm{m})]$} \\
\hline$\eta$ & Room charge density & {$\left[\mathrm{C} / \mathrm{m}^{3}\right]$}
\end{tabular}

\section{References}

[1] A Klinkenberg and J L van der Minne. Electrostatics in the Petroleum Industry. Elsevier, Amsterdam, 1958.

[2] H Helmholtz. Studien über electrische Grenzschichten. Analen der Physik und Chemie, Neue Folge 7: 337-382, 1879.

[3] P Fung et al. A Nonlinear Corrosion Double-Layer Model for Laminar Flow Electrification of Hydrocarbon Liquids in Long Metal Pipes. Journal of Electrostatics, 1997.

[4] N N Hydac. Schwerwiegende Folgen vermeiden! Ölhydraulik und Pneumatik O+P, 3/2012: 20-23, 2012.

[5] G Lütgens. Statische Elektrizität begreifen beherrschen - anwenden. ExpertVerlag, Renningen, 2005 .

[6] A Sasaki and S Uchiyama. Generation of Static Electricity During Oil Filtration. Lubrication Engineering, Vol. 55, No. 9: 14-21, 1999.

[7] A Bourgeois et al. Flow Electrification in Power Transformers: Study of a Potential Remedy. IEEE Transactions on Dielectrics and Electrical Insulation, Vol. 13, No. 3, 650-656, 2006.

[8] A P Washabaugh and M Zahn. A Chemical Reactionbased Boundary Condition for Flow Electrification. IEEE Transaction on Dielectrics and Electrical Insulation, Vol. 4, No. 6, 688-709, 1997.

[9] A Küchler. Hochspannungstechnik Grundlagen Technologie - Anwendung. Springer, Berlin, 2009.

[10] H Chmela and R Smetana. Feldmühle - Ein Messgerät für elektrische Felder.

http://www.hcrs.at/FELDMU.HTM, visited October 12, 2012.

[11]N N. Feldmühle. http://www.rapp-instruments.de/staticmachines/measuring/feldmuehle/feldmuehle.htm, visited October 12, 2012.

[12] T V Oommen and E M Petrie. Electrostatic Charging Tendency of Transformer Oils. IEEE Transactions on 
Power Apparatus and Systems, Vol. 103, No. 7, 19231931, 1984.

[13]K R Stonitsch. Einfluß der hydraulischen Strömungsverhältnisse auf die elektrostatische Aufladung der Kühlkanäle von HochspannungsLeistungstransformatoren. Dissertation, TU Graz, 1995.

[14] A J Morin et al. An Absolute Charge Sensor for Fluid Electrification Measurements. IEEE Transactions on Electrical Insulation, Vol. 26, No. 2, 181-199, 1991.

[15] J K Nelson and M J Lee. Tandem-chamber Charge Density Monitor. IEEE Transactions on Electrical Insulation. Vol. 25, No. 2, 399-404, 1990.

[16]H Haken and H C Wolf. Molekülphysik und Quantenchemie. Springer, Berlin, 2006.

[17] N N. Vermeidung von Zündgefahren infolge elektrostatischer Aufladungen. TRBS 2153, 2009.

[18] O Weimann. Die Abscheidung von Lufblasen aus Schmierölen durch konstruktive Maßnahmen. Dissertation, TU Darmstadt, 1971. 